\title{
INTRAMEDULLARY SPINAL CORD METASTASIS FROM MAMMARY CARCINOMA
}

\author{
By F. L. Mastaglia, M.B., M.R.A.C.P., M.R.C.P. and \\ B. A. Kakulas, M.D., M.R.A.C.P., M.C.P.A., M.C.Path. \\ From the Department of Pathology, University of Western Australia and the \\ Neuropathology Laboratory of the Royal Perth Hospital
}

Metastasis of malignant tumours to the substance of the spinal cord is generally considered to be rare. Since the first recorded case of metastases from mammary carcinoma by Buchholz (I897) only 39 other cases have been reported in the literature. Benson (1960) reviewed 24 cases and added three of his own from bronchogenic carcinoma. Chason, Walker and Landers (1963) reported ten further cases and Sherbourne, Tribe and Varma (I964) reported another three cases. Metastases to the cord are almost certainly commoner than these figures indicate for, as Willis (1952) has pointed out, the spinal cord is seldom examined in a routine necropsy unless there are clinical manifestations of spinal cord involvement during life. Chason's figures provide a truer estimate of the incidence of metastatic carcinoma in the spinal cord. In a series of 1096 patients dying of carcinoma, the spinal cord was examined in every case and intramedullary metastases were found in ten cases, an incidence of I per cent. Of the 200 cases in their series with central nervous system metastases, 5 per cent. had spinal cord metastases.

The present report is of metastasis to the thoracic cord causing paraplegia eight years after radical mastectomy for carcinoma of the breast.

Case Report. A 47-year-old woman developed carcinoma of the left breast in 1959 and a radical mastectomy was performed. She remained in good health until May 1967, when she developed dyspnoea on exertion. In July 1967, following an episode of herpes zoster involving the fourth thoracic dermatome on the right side she developed numbness and tingling and weakness in the lower limbs and over the course of the next week became confined to bed.

On examination she was wasted, dyspnoeic and cyanosed. She was completely paraplegic with spasticity in the lower limbs. The tendon jerks were increased in the lower limbs and both plantar responses were extensor. There was complete loss of all sensory modalities below the level of the fourth thoracic dermatome on both sides. Muscle power and tone were normal in the upper limbs and there was no sensory loss. The pulse rate was 140 per minute and the blood pressure $130 / 85 \mathrm{~mm}$. $\mathrm{Hg}$. The jugular venous pressure was elevated $6 \mathrm{~cm}$., the liver was enlarged $6 \mathrm{~cm}$. below the right costal margin and there were large pleural effusions. Radiographs of the spine showed multiple osteosclerotic deposits in the vertebral bodies with collapse of the body of $\mathrm{T}_{7}$.

A course of deep X-ray therapy was administered to the thoracic spine with no improvement in the patient's neurological state. The pleural effusions were aspirated on several occasions for relief of dyspnoea and radio-active gold was injected into the left pleural cavity. Her condition deteriorated steadily and she died nine weeks after first developing signs of spinal cord involvement.

Necropsy Report. The body was that of a wasted middle-aged woman. Telangiectases were present over the left upper chest and there was lymphoedema of the left arm. There was no evidence either of local recurrence of the tumour or of metastases in the 
axillary lymph nodes. However, there was widespread metastatic dissemination of tumour involving both pleural cavities, the left lung, pericardium, adrenals, ovaries and uterus and, in addition, multiple osseous deposits were present in the skull, ribs and throughout the vertebral bodies. The lower thoracic and lumbar vertebrae were most severely affected and there was a compression fracture of the body of the seventh thoracic vertebra. There was, however, no evidence of spinal cord compression at this level and the dura was not involved by tumour at any level. Histological examination of the tumour deposits showed poorly differentiated adenocarcinoma. In most of the metastatic sites, nests of neoplastic cells were associated with a dense fibroblastic stromal reaction and there was extensive lymphatic infiltration.

Neuropathology Findings. The whole spinal cord was removed together with its membranes. The dura and subdural space were normal and showed no evidence of involvement by tumour. On opening the dura it was found that the spinal cord was abnormal in the mid-thoracic region from the level of $\mathrm{T}_{3}$ to $\mathrm{T} 6$. In this region the cord was slightly expanded and its surface presented a corrugated nodular appearance (fig. I). Sections at intervals of approximately $0.5 \mathrm{~cm}$. were placed throughout this abnormal area. At the level of $\mathrm{T}_{4}$ a grey semi-opaque roughly circular lesion with a paler border was present in the central part of the cord. The lesion was largest at the level of $\mathrm{T}_{4}$, where it measured $8 \mathrm{~mm}$. in diameter, and extended from $\mathrm{T}_{3}$ to $\mathrm{T}_{5}$ for a distance of $3.5 \mathrm{~cm}$., occupying a similar position in the cord and tapering in its upper and lower portions. The cord immediately above the level of the lesion showed a complete loss of normal anatomical markings particularly in its posterior half. At the level of $\mathrm{T}_{5}$ there was a slit-shaped lesion $3 \mathrm{~mm}$. in length adjacent to the right posterior grey horn with orange haemosiderin staining of its walls. At T6, the normal anatomical markings of the cord were indistinct and there were multiple peripheral areas of softening (fig. 3). Sections through the cervical cord showed pallor of the most medial part of the fasciculus gracilis on both sides. Sections through the lumbar cord showed pallor in the posterior parts of the lateral columns at the site of the cortico-spinal tracts. The only other metastasis in the central nervous system was in the white matter of the right cerebellar hemisphere and measured $\mathrm{I} \mathrm{cm}$. in diameter.

Histological examination of the spinal cord at the level of the lesion (fig. 2) showed a deposit of tumour in the central part of the cord completely obliterating the grey matter. The central part of the lesion was acellular and hyaline and tumour cells were present only in the peripheral portions where they were arranged in sheets and in places tended to form glandular structures. The tumour cells were moderately pleomorphic and mitotic figures were present. Posteriorly the tumour extended almost to the surface of the cord and extended into the dorsal root on the right side (fig. 2). The white matter in the immediate vicinity of the lesion showed spongy change and fibrous astrocytic proliferation. At the level of $\mathrm{T}_{3}$, there were two small cavitated peripheral areas of necrosis in the posterior portion of the lateral columns containing large numbers of foamy macrophages. In addition, there was marked vacuolation and fibre loss in the posterior white columns with pronounced fibrous gliosis and many pleomorphic microglia and foamy macrophages. Small vessels were prominent and the dorsal nerve roots were congested and gliotic. Many anterior horn cells were swollen and showed indistinct nuclear markings. The slit-shaped lesion at the level of $\mathrm{T}_{5}$ was found to be an area of past haemorrhage containing many haemosiderin-laden macrophages. At T6 there were multiple peripheral areas of softening, particularly in the lateral columns. The lesions involved the peripheral portions of the white matter bordering on the surface of the cord and showed destruction of white matter with many axonal swellings and foamy macrophages and small numbers of fibrous astrocytes. The ventral nerve roots at this level also showed axonal swelling and fragmentation and foamy macrophages were present. Sections from the cervical cord showed loss of myelinated fibres in the medial portion of the fasciculus gracilis and in the spinothalamic tracts. In these regions, axons showed swelling and fragmentation, and foamy macrophages and fibrous astrocytes were present. Sections from the lumbar 


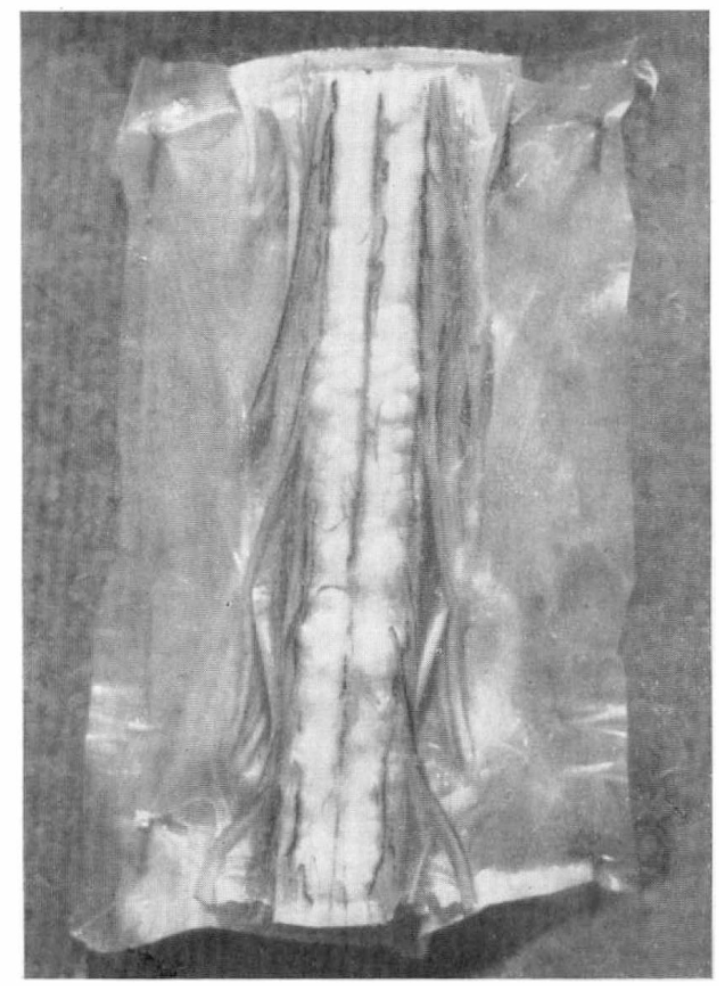

FIG. I

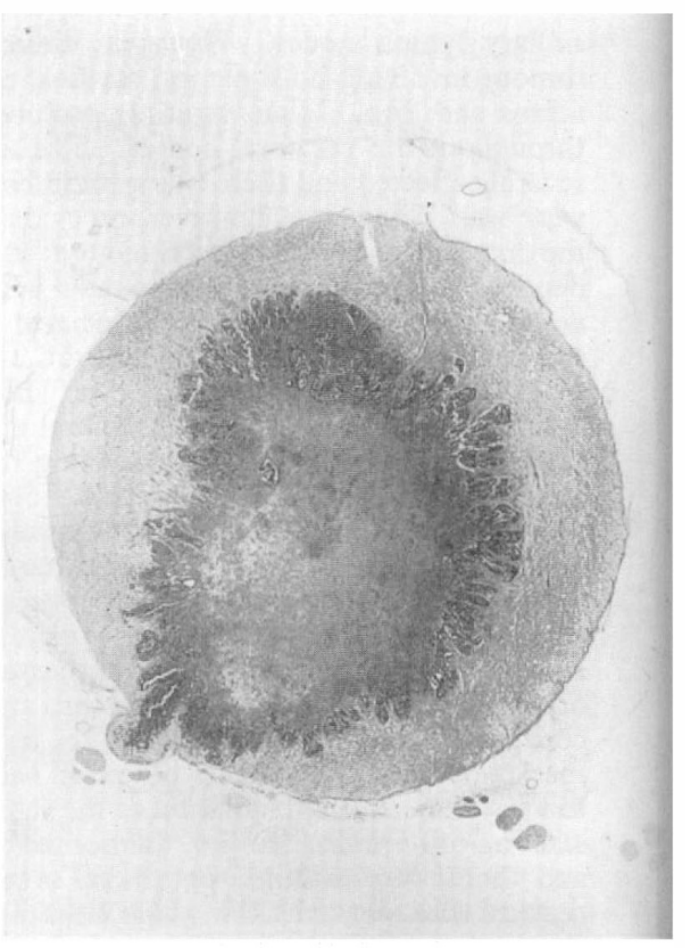

FIG. 2

Fig. I.-Thoracic cord showing expansion and irregular nodularity.

Fig. 2.-Cross-section of spinal cord at $\mathrm{T}_{4}$ showing central tumour deposit extending into dorsal nerve root. Haematoxylin and eosin. $\times 5.6$.

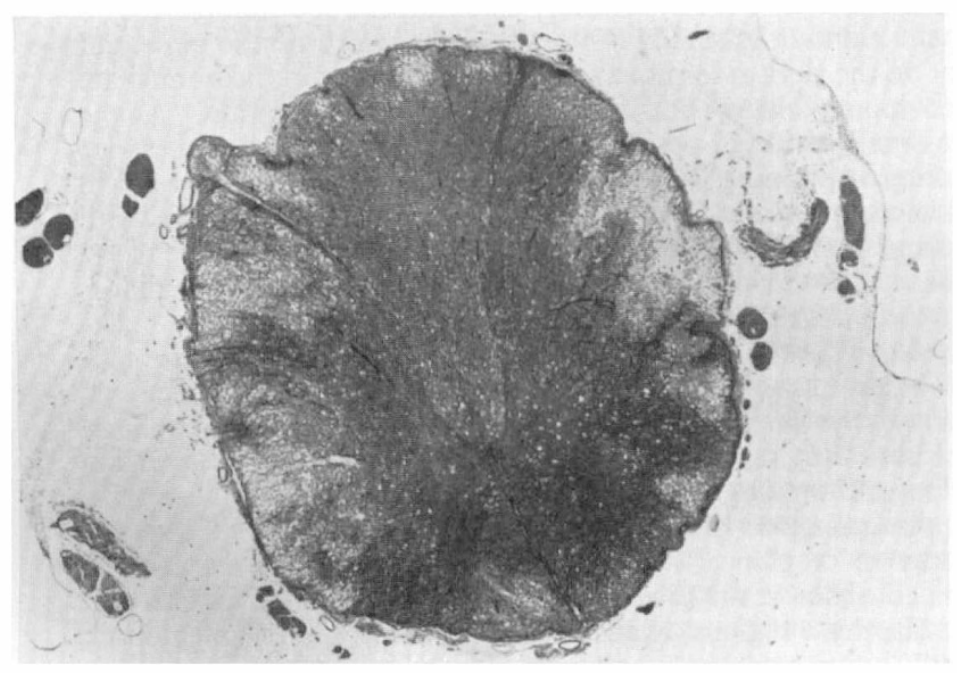

FIG. 3
Fig. 3.-Cross-section of spinal cord at T6 showing multiple peripheral areas of softening. Haematoxylin and eosin. $\times 6 \cdot 35$. 
cord showed similar changes in the cortico-spinal tracts. Myelin stains showed a reduction in numbers of myelinated fibres in the fasciculus gracilis and spinothalamic tracts above the level of the lesion and in the cortico-spinal tracts below the level of the lesion. Oil red O-stains showed considerable neutral fat in macrophages in these areas.

\section{DISCUSSION}

The patient remained well for eight years after removal of an adenocarcinoma of the breast. Nine weeks before her death she developed symptoms of thoracic cord involvement and over the course of a week became completely paraplegic with a sensory level at $\mathrm{T}_{4}$. An initial diagnosis of spinal cord compression due to vertebral or dural metastases was made. In addition, there was pericardial and pleural involvement with congestive cardiac failure and pleural effusions. Her condition deteriorated steadily despite X-ray therapy to the spine and instillation of radio-active gold into the left pleural cavity. At necropsy there was no evidence of recurrence of the primary tumour but metastases were widespread. Apart from a single metastasis in the cerebellum the only other central nervous system deposit was in the thoracic cord extending from $\mathrm{T}_{3}$ to $\mathrm{T}_{5}$. The tumour extended into the right dorsal nerve root at the level of $\mathrm{T}_{4}$ and was associated with multifocal myelomalacia below the lesion, gliosis of the cord above the lesion and secondary ascending and descending long-tract degeneration. The finding of destructive lesions in the cord, of fibrous gliosis and of secondary-tract degeneration is consistent with the nine-week interval between the onset of symptoms of cord involvement and death.

Over half of the 40 previously reported cases of spinal cord metastases have been from a bronchogenic primary and only three other cases of metastases from breast carcinoma have been reported (Buchholz, I897; Hofheinz, I909; Amyot \& Laurin, 1939). The thoracic cord has been most frequently involved, as in the present case. In most cases the metastases have been multiple and there are few reports of single sharply defined deposits (Gaylor \& Howie, I938; Rasch, Rosenbaum \& Heimburger, I953; Sherbourne et al., I964). Most cases have also had associated cerebral metastases. The deposit is commonly surrounded by an area of oedema and glial reaction and this, together with the space-taking effect of the lesion, leads to expansion of the cord and is probably responsible for the peculiar corrugated appearance of the cord in the present case. Ischaemic lesions of contiguous areas of the cord above and below the lesion are common (Benson, I960) and probably result from vascular insufficiency due to compression of vessels, particularly in the thoracic region where the spinal canal is narrowest. Vascular insufficiency may account for the relatively abrupt clinical presentation in some cases.

Benson (1960) has drawn attention to the rather consistent clinical picture of intramedullary metastases and to the more rapid onset than that of most primary spinal cord tumours. Herpes zoster is a recognised complication in malignant disease of the spine and has been attributed to activation of a latent virus by tumour involvement of the dorsal root ganglia (Merritt, 1967). It is of interest that in the present case, although the dorsal root ganglia were not involved, the fourth dorsal nerve root was invaded by tumour. The diagnosis of intramedullary metastases has rarely been made during life, the chief differential diagnosis being from extramedullary metastases. Pain, both vertebral and radicular, is usually a prominent 
feature of extra-medullary metastases and this, together with findings on myelography should allow differentiation. Other less likely conditions to be considered in a patient with known malignancy include subacute necrotic myelopathy (Mancall \& Rosales, 1964) and radiation myelopathy when deep X-ray therapy has been given either to the primary or to metastases. When cord metastases precede clinical detection of the primary growth, diagnosis is most difficult and other forms of subacute progressive myelopathy must also be excluded.

There are several routes by which malignant tumours may spread to the substance of the spinal cord. Haematogenous metastasis is probably of greatest importance, particularly when there is widespread dissemination of the tumour throughout the body as in the present case. The rarity of intramedullary metastases in comparison to extradural spinal and intracerebral metastases is almost certainly related to regional vascular factors. It has been suggested that although the spinal cord has a rich vascular supply most of the communicating collateral vessels are of small calibre and may be less accessible to tumour cells in the blood stream (Benson, I960). Involvement of the cord may also result from seeding through the vertebral system of veins, spread through the subarachnoid space or by direct involvement of the cord by dural deposits of tumour.

\section{SUMMARY}

Metastases to the substance of the spinal cord are almost certainly more frequent than generally supposed. The majority of previously reported cases have been bronchogenic primaries and only three have been from mammary carcinomas. The case of a 47-year-old woman who became paraplegic eight years after radical mastectomy for carcinoma of the breast is reported. An episode of herpes zoster in the fourth thoracic dermatome preceded the onset of paraplegia. At necropsy there were widespread metastases in many organs. A solitary metastasis was found in the thoracic cord extending from $\mathrm{T}_{3}$ to $\mathrm{T} 6$ and extending into a dorsal nerve root at $\mathrm{T}_{4}$.

We would like to thank Mr. H. Stewart and Mr. G. M. Bedbrook for clinical information and permission to report the case. Mr. H. Upenieks, A.R.P.S. prepared the photomicrographs.

Dr. F. L. Mastaglia was in receipt of a Saw Medical Research Fellowship from the University of Western Australia.

\section{REFERENCES}

Amyot, R. \& LaURin, P. W. (1939). Presse médicale, 47, 978-980.

BENSON, D. F. (1960). Neurology, Minneapolis, I0, 28 I-287.

BuchHolz, F. (1897). Monatsschrift für Psychiatrie und Neurologie, 4, 183-190.

Chason, J. L., Walker, F. B. \& Landers, J. W. (1963). Cancer, 16, 78 I-787.

GaYlor, J. B. \& HowIE, J. W. (1938). Fournal of Neurology and Psychiatry, 1, 30I-305.

HofHeInZ, G. (1909). Deutsche Zeitschrift für Nervenheilkunde, I I 7-I I 9, 226-235.

MANCALl, E. L. \& Rosales, R. K. (I964). Brain, 87, 639-656.

MerRitT, H. H. (1967). A Textbook of Neurology, p. 69. Philadelphia: Lea and Febiger.

Rasch, G. C., Rosenbaum, D. \& Heimburger, R. F. (I953). Neurology, Minneapolis, 3, 3II-3I5.

Sherbourne, D. H., Tribe, C. R. \& Varma, S. (1964). Paraplegia, 2, IOO-I II.

WILlIS, R. A. (I952). The Spread of Tumours in the Human Body, pp. 292-293. London: Butterworth. 